\title{
Décrire les migrations internationales
}

Les expériences de la co-présence

William Berthomière et Marie-Antoinette Hily

\section{CpenEdition}

Journals

Édition électronique

URL : https://journals.openedition.org/remi/2819

DOI : $10.4000 /$ remi.2819

ISSN : $1777-5418$

Éditeur

Université de Poitiers

Édition imprimée

Date de publication : 1 juillet 2006

Pagination : 67-82

ISBN : 2-911627-42-3

ISSN : 0765-0752

Référence électronique

William Berthomière et Marie-Antoinette Hily, " Décrire les migrations internationales », Revue européenne des migrations internationales [En ligne], vol. 22 - n² 2 | 2006, mis en ligne le 01 juin 2009, consulté le 14 avril 2022. URL : http://journals.openedition.org/remi/2819 ; DOI : https://doi.org/ 10.4000/remi.2819

Ce document a été généré automatiquement le 14 avril 2022.

(C) Université de Poitiers 


\section{Décrire les migrations internationales}

Les expériences de la co-présence

William Berthomière et Marie-Antoinette Hily

1 De New York à Perth ou de Pékin à Buenos Aires, l'ensemble de la planète est aujourd'hui concerné par les migrations internationales. Estimés à plus de 200 millions de personnes ${ }^{1}$, les migrants internationaux ont donc contribué à forger une représentation du monde que résume le mot de "globalisation". Même si le choix d'émigrer est loin d'être accessible à tous, aujourd'hui, la migration n'est plus l'« apanage » des pays du Nord comme elle l'était encore au cours des années cinquante (Simon, 1995). Depuis, la situation s'est progressivement inversée et les pays du Nord ont tous accueilli des contingents de migrants internationaux décrits classiquement dans la terminologie des facteurs push and pull: accords internationaux de maind'œuvre, développement des multinationales, acquisition/valorisation de compétences, inégale répartition des richesses, exodes liés aux conflits armés ou aux dégradations de l'environnement, trafic d'êtres humains ou bien encore nécessité d'assurer un avenir meilleur aux enfants (Stalker, 2001).

2 Parallèlement à ce "renversement des flux migratoires", des migrations se sont développées et densifiées, dans des pays en voie de développement. Qualifiées de migrations sud-sud, ces mobilités reposent sur des systèmes d'échanges régionaux, souvent anciens, auxquels sont venus s'ajouter, plus récemment, des flux migratoires internationaux impulsés notamment par les mesures restrictives à l'immigration imposées par les États du Nord. Ainsi, tandis que la méconnaissance des dynamiques migratoires internationales provoque dans les pays développés des réactions de crainte d'une invasion migratoire en provenance des pays du Sud, c'est pourtant entre ces pays que l'on enregistre, aujourd'hui, la majorité des flux, constitués massivement de mouvements de réfugiés. Sur l'ensemble de la planète, ces migrants, au sens du Haut Commissariat pour les Réfugiés des Nations Unies (HCR), et ceux définis sous le terme d'« éco-réfugiés $»^{2}$ seraient estimés à environ 40 millions d'êtres humains (LasaillyJacob, 1999). 
3 En ce début de $\mathrm{xxl}^{\mathrm{e}}$ siècle, le panorama migratoire international dessine deux grandes dynamiques migratoires aux orientations sud-nord et sud-sud mais, surtout, confirme que la quasi-totalité des pays sont concernés par la «mondialisation migratoire». Les grands systèmes migratoires de la planète, que sont l'Amérique du Nord, l'Europe occidentale et le Japon, accueillent aujourd'hui des migrants dits « au long cours », des personnes dont l'espace de vie s'organise entre des pays très éloignés.

4 C'est ce rapport espace-temps que la production scientifique sur la mondialisation a cherché à saisir. Différentes étapes de la mise en théorie de cette multiplicité des formes de la mobilité ont ainsi été franchies au cours des cinquante dernières années en tentant d'articuler poursuite de la connaissance des formes sociales et analyse de l'exercice du politique. La critique principale qu'a affronté la théorisation des migrations internationales a principalement reposé sur ce point de tension en faisant de la mondialisation, pour les uns, une nouvelle forme d'être-au-monde, pour les autres, un objet sans réelle portée heuristique puisqu'il demeure contraint par la domination du modèle État-nation.

5 La complexité de la nature des mobilités humaines et bien plus encore leur internationalisation ont enrichi le lexique de termes permettant de penser ces processus et phénomènes contemporains. Ainsi, circulation, champ ou territoire migratoire, transnationalisme, société en réseaux ou encore "glocalisation " sont des notions largement utilisées pour décrire les reconfigurations dans l'espace et dans le temps de nos sociétés. Mais si l'ensemble de ces notions ont largement contribué au développement de la recherche, on peut se demander si elles ne tendent pas à épuiser nos représentations.

6 La réflexion ces dernières années a également cherché à démêler la production des identités et des appartenances culturelles dans des sociétés marquées par la coprésence de populations diverses par leurs origines. Dès lors le débat scientifique influencé par les sciences politiques n'a cessé d'osciller entre des interrogations dépendantes d'un modèle stato-national et une approche qui relève d'une sociologie des migrations qui s'est efforcée de questionner le rapport espace-temps dans les analyses.

7 L'objectif de cet article est de rendre compte d'une part des usages des notions qui ont été mobilisées pour penser les formes migratoires mondialisées, en rupture avec les approches développées jusque dans les années 1970 et d'autre part de privilégier un point de vue qui relève d'un mode d'interrogation qui ne désigne pas tant les causes des migrations ou les motivations à migrer et les transformations qu'elles induisent que d'accéder à une compréhension plus modeste des modes d'organisation des collectifs en co-présence et la façon dont ils saisissent des "occasions", là où les gens se rencontrent, dans des espaces de sociabilité non figés: la rue d'Arménie dans le quartier de Bourj-Hammoud à l'est de Beyrouth et le quartier de la gare routière à Tel Aviv.

\section{Circulation migratoire et notions contemporaines}

8 La distance progressive vis-à-vis des problématiques développées dans les années 1970 s'est opérée notamment avec l'introduction de la notion de circulation migratoire forgée pour décrire la complexité croissante des phénomènes de mobilité internationale (Hily, 
1998, 2003). Elle fait suite à celles de noria, de champ et d'espaces migratoires, travaillées par des chercheurs en majorité issus de la géographie et dont l'objectif était de dépasser la dualité des approches qui portaient soit sur les sociétés d'installation, organisées autour des problématiques de l'intégration, soit sur les pays d'origine centrées sur celles du développement ${ }^{3}$.

9 La notion de circulation migratoire correspond à une démarche qui tente de tenir compte à la fois des espaces concernés par les migrations, des déplacements accrus des personnes entre différents lieux et des flux matériels (biens, services, remises) et idéels (normes, valeurs, représentations) induits par les migrations. Le migrant et le circulant, plutôt que l'immigré ou l'émigré, deviennent des figures centrales de l'activité de recherches et l'attention est portée sur les pratiques et sur les initiatives des personnes, sur les itinéraires et les espaces parcourus.

10 Sous le terme englobant de circulation migratoire il s'agit donc pour les chercheurs d'intégrer dans les analyses ce qu'ils considèrent comme des nouvelles formes de mobilités où les migrants et les migrantes semblent s'émanciper des normes du salariat pour s'inscrire dans des activités économiques formelles et informelles portées par des réseaux socio-économiques plus ou moins structurés. Ce sont alors ces dispositifs commerciaux qui s'observent sur des espaces transnationaux qui intéressent les analyses ${ }^{4}$. Dans ces démarches, la notion de projet migratoire devient centrale pour comprendre les logiques "d'être en relation » et "de nouer des relations » dans un espace de circulation où s'exerce le jeu entre territoires des ancrages et territoires des circulations.

11 En renonçant à expliquer les migrations par les déterminants structuraux, les problématiques de recherche ont érigé en objet privilégié de l'enquête, les ferments des collectifs migrants en interrogeant les connexions et les échanges. Les modes d'organisation des migrants sont conçus comme des produits plus ou moins instables de ce processus. L'accent est mis sur les réaménagements constants selon les contraintes. Les manières d'agir dans l'action (ruser, marchander etc.) ou les attitudes (confiance, engagement, donner sa parole) sont analysées en privilégiant les interactions. Les recherches mettent aussi l'accent sur les apprentissages (c'est-à-dire apprendre les règles en usage dans les milieux traversés), sur les compétences à circuler (c'est-à-dire déjouer les dispositifs de contrôle; compter sur des relations, saisir les occasions). Elles mettent ainsi en avant différentes théories de la mobilisation des ressources: économies des coups (Schmoll, 2004), économies d'opportunité (Peraldi, 2001; Manry, 2001), économies informelles (Tarrius, 2002) et économie des transports (de Tapia, 1996). Ces approches multiformes du lien social ont été autant de manières d'interroger les collectifs au prisme de la notion de savoir (savoir-faire bien sûr mais aussi savoir-circuler ou bien encore savoir-séjourner) prise pour objet afin de dégager les capacités d'entreprendre du migrant.

12 Dans une perspective plus ethno-culturelle, la lecture reprend celle « des cultures de la mobilité " (notamment sous l'impulsion de James Clifford) et la création de microespaces sociaux d'autonomie. Dans ces processus, le «migrant » - et ses performances - devient l'acteur crucial saisi sous différentes figures : le colporteur, le commerçantambulant, la nana benz, l'aventurier et enfin le nomade. Cette posture a largement bénéficié de l'ensemble de la mise en problématique entreprise dans le cadre de la recherche sur les diasporas, comme modèle permettant une lecture du rapport à l'espace-temps. 
13 Les recherches sur la «diaspora » se sont inspirées d'une littérature philosophique laissant place à la notion d'hybridité employée par les auteurs «post-modernes » dans la proximité avec celles de métissage et de créolisation. Les études sur la Caraïbe sont en cela exemplaires avec l'utilisation de la notion de rhizome (forgée par Deleuze et Guattari) dans les travaux d'Édouard Glissant (Chivallon, 1997). Toujours dans ce champ d'analyses, un pas supplémentaire dans la réflexion a été franchi avec le travail de James Clifford (1994) proposant le terme de «traveling cultures » et le débat suscité par la publication de The Black Atlantic de Paul Gilroy (1993). Ces auteurs, comme également Homi Bhabha, Stuart Hall ou Arjun Appadurai, ajoutent une dimension métaphorique au terme de diaspora qui devient synonyme pour eux des déracinements de la condition post-coloniale. Robin Cohen (1997a) a résumé ce temps de la recherche en soulignant que les "diasporas are positioned somewhere between "nations-states" and "traveling cultures" in that they involve dwelling in a nation-state in a physical sense, but traveling in an astral or spiritual sense that falls outside the nation-state's space/time zone ».

La recherche sur la diaspora s'organise dans un double mouvement, celui impulsé par les représentants « du tournant culturel » et celui soutenu porté par des chercheurs interrogeant la relation au politique dans la dialectique « diaspora versus État-Nation ». Dans ce double mouvement, la notion d'État-Nation se résume alors, selon Paul Gilroy (1994), comme une notion politique qui clôt la dispersion puisqu'elle sous-entend in fine, d'un côté l'assimilation, de l'autre, le retour.

15 Rompant avec la métaphore culturelle développée sur la dualité pays rêvé/pays réel pour reprendre Édouard Glissant, l'approche en termes de transnationalisme a posé de plain-pied la théorisation dans le champ du politique, même si la critique qu'ont dû affronter ces promoteurs fut celle d'une déconnexion d'avec le cadre stato-centré de nos sociétés (Waldinger, 2004).

16 Établi en rupture avec une approche "classique» de l'État-Nation défini dans les termes d'un peuple partageant une culture commune au sein d'un territoire aux limites finies (bounded territory), ce nouveau mode de penser le territoire (E. Ma Mung, 1998) et l'État-Nation propose une citoyenneté intégrant «ceux qui vivent physiquement dispersés à l'intérieur des frontières de nombreux autres États mais qui participent socialement, politiquement, culturellement et souvent économiquement à l'État-Nation de leurs origines» (Basch et al., 1994). Ces contributions à la réflexion pour des auteurs comme James Clifford au lieu de clarifier le débat l'ont rendu un peu plus confus du fait " $d u$ désordre de termes descriptifs et interprétatifs qui se bousculent et convergent pour caractériser la zone de contacts entre nation, culture et espace (région)» (1994).

Les "études transnationales" se sont installées dans le paysage de la recherche en proposant de tourner la page avec la représentation classique des recherches, espace d'origine et/ou espace d'installation, qui enferme dans des catégories duales. Le " champ transnational » a acquis une légitimité après qu'un courant de l'anthropologie se soit dégagé des théories de la modernisation et d'une perspective structuraliste privilégiant une approche macro-historique des phénomènes (Monsutti, 2004). Dans la littérature anglo-saxonne, les recherches s'ouvrent alors sur une interrogation classique, déjà conceptualisée en France avec les travaux d'Abdelmalek Sayad, en opérant un continuum entre les sociétés d'origine et d'accueil "qui ne forment plus qu'une seule et même arène de l'action sociale " (Brettell, 2000 : 104-105). Cependant malgré les nombreux travaux empiriques menés dans cette direction, la théorie du transnationalisme reste discutée. Et comme a pu le souligner Alessandro Monsutti 
(2004) la question majeure qu'elle doit affronter est de savoir si elle s'articule sur l'objet d'étude ou sur le regard qu'elle lui porte. En se définissant comme une approche inédite d'un phénomène dit nouveau, les artisans du transnationalisme - principalement Nina Glick Schiller et ses collègues - se sont vus opposer le rappel de la profondeur historique et séculaire du phénomène de globalisation et d'identification des individus à plus d'une communauté (Monsutti, 2004). Le débat fut alors ramené à un questionnement sur l'ampleur du phénomène observé bien plus que sur sa nature reprenant en cela le double écueil à éviter selon Boyd: "an undersocialized view of migration in which all action reflected individual wishes and preferences" et "an oversocialized view in which people were passive agents in the migratory process " (Monsutti, $2004: 46)$.

18 Nina Glick Schiller et Peggy Levitt ont très justement rappelé qu'elles n'ignoraient pas l'abondante production qui a traité de manière discrète du transnationalisme depuis la publication de l'article Transnational America par Ralph Bourne en 1916, tout en insistant sur la légitimité de leur point de vue qui permet aujourd'hui de s'émanciper de la « container perspective » qui a dominé les sciences sociales jusqu'à récemment avec la référence stato-centrée de l'intégration (Glick Schiller, 2006). Les résultats des "transnational migration studies" ont été largement confortés par une réflexion conceptuelle (Portes, 2001) et méthodologique sur les relations entre processus de construction nationale et migration, un des aspects privilégiés de la démarche visant à comprendre comment a pu s'exercer la fixation « of a scientific eye to the body of the nation came about and how this relationship has evolved through different phases of nation building» (Wimmer 2002 : 325).

Les nombreux ajustements et clarifications qui ont permis le débat sur la notion de transnationalisme, n'ont cependant pas dissipé le caractère insaisissable des situations qui s'offrent à l'analyste de la mondialisation migratoire. La difficulté à appréhender les processus sociaux à l'œuvre - qu'ils soient définis en termes de stratégies, de réseaux ou encore d'échelles - repose avant tout sur le constat que les conceptualisations proposées n'ont su trouver les attributs d'une approche déliée de normativité. En cela la critique est sévère, mais elle donne matière à réflexions. D'une part, comment sortir de cette impasse qui semble n'offrir aucune issue possible face à une lecture causale où les déterminants sociaux, économiques et politiques dominent, d'autre part, une des voies possibles n'est-elle pas d'opter pour des méthodes qui relèvent d'une sociologie de l'action? On rappellera que James Clifford avait de privilégier le terrain comme "une expérience de décentration qui n'est plus conçue en termes uniquement spatiaux » dans l'idée que «les communautés diasporiques ne peuvent être réduites à un épiphénomène de l'État-nation ou du capitalisme global, car elles représentent une caractéristique constitutive du monde contemporain » (Monsutti, $2004: 42$ ).

Le débat conduit l'analyse à s'orienter vers la description et à envisager le lien social comme l'écrivait Simmel «sur la base de relations objectives, c'est-à-dire situées dans l'être des sujets » (Simmel, 1999: 409). Une telle approche se démarque de celle qui interroge le local et le global à la manière de Ulf Hannerz : « an arena where various people's habitats intersect, and where the global, or what has been local somewhere else, also has some chance of making itself at home » (1996: 28). Sa proposition fait du lieu l'espace de la description des situations dans leur singularité et leur immédiateté, c'est une démarche possible. Notre approche est différente en ce sens qu'elle n'a pas pour objectif l'explication. 


\section{Les « occasions sociales " de la mise en presence : bourj-hammoud-neve sha'anan}

21 Les collectifs en situations d'interactions multiplient les "occasions sociales " pour reprendre une terminologie goffmanienne (Giddens, 2005). Nous adhérons à cette approche qui installe un point de vue différent de l'approche historique et structurale pour plus modestement décrire ces situations inscrites dans la forme de l'État-nation mais que les collectifs réinterrogent par leur présence. Ce parti pris permet de rendre compte des pratiques ordinaires de migrants qui s'offrent à l'observation là où ils sont devenus visibles. Nos exemples ont été choisis volontairement dans des villes du Moyen-Orient, Beyrouth et Tel Aviv où depuis une dizaine d'années des migrants ont acquis une visibilité. C'est cette visibilité que nous interrogeons comme un «événement » dans les routines des citadins.

\section{La construction d'une " banlieue » beyrouthine, Bourj-Hammoud : des étrangers actifs et sous contrôle}

Présentée comme « le grand marché de Beyrouth », Bourj-Hammoud, banlieue est de la capitale accueille, selon des estimations non officielles, un millier de migrants SriLankais, Philippins, Bangladais, Indiens Tamouls, Éthiopiens, Égyptiens, Ghanéens, Syriens, Soudanais, Kurdes et Béninois. Souvent en situation irrégulière, soit qu'elles sont arrivées avec un permis de travail arrivé à expiration, soit qu'elles se présentent comme réfugiés, demandeurs d'asiles, exilés etc., ces personnes ont trouvé dans cet espace urbain aux immeubles à plusieurs étages et à l'habitat serré et dense, qui ne dénotent apparemment ni l'aisance ni la misère, des occasions, qu'elles paient chèrement, de se loger dans des appartements délabrés et mal entretenus. Certaines travaillent dans le quartier, d'autres s'emploient comme « servantes » dans les familles aisées beyroutines ou dans le bâtiment ou encore dans la restauration-hôtellerie. Ces nouveaux « résidents » vivent dans la précarité, dans la peur du contrôle. Et pourtant c'est dans cette incertitude quotidienne que s'organise une vie sociale avec l'espoir de "s'en sortir» ou plus justement de trouver des moyens de gagner de l'argent, contraints qu'ils sont d'accepter des conditions de travail difficiles et des emplois mal rémunérés. Ici les «migrants» et particulièrement les «migrantes" s'activent à conjuguer nécessité de la discrétion et de l'évitement et rencontre avec les «locaux/ nationaux", ceux qui se revendiquent comme Libanais, Arméniens ou Libanoarméniens.

\section{Migrations et activités du commerce}

$\mathrm{Au}$ début $\mathrm{du} \mathrm{xx}^{\mathrm{e}}$ siècle Bourj Hammoud, village agricole va progressivement se transformer avec et après l'arrivée des Arméniens.

C'est sous le mandat français que trois vagues de réfugiés arméniens (1918, 1922 et 1939) venant respectivement de l'Arménie, de la Turquie et de Sandjak d'Alexandrette bâtirent initialement ce qu'ils appellent "la petite Arménie " et que nombre de Libanais du quartier appellent encore «le camp St Jacques». Aux ruelles étroites bordées de petites maisons en dur, blanchies à la chaux, ce qui reste de cet îlot dans le 
quartier abrite encore de vieilles familles arméniennes aux faibles ressources. Certains travaillent le cuivre et tentent de maintenir un artisanat arménien.

La population connaît un taux élevé de natalité, un faible niveau de vie et une grande diversité culturelle. Le père Mécérain écrit en 1972: «Avec des planches de fortune on fabriqua des baraques... une cité ouvrière se forma, spontanément avec toutes sortes de métiers. Les femmes qui le pouvaient allaient travailler en ville. Les jeunes filles s'usaient les prunelles à broder, à faire des dentelles contre une rétribution journalière très modique» (Buccianti, 1972).

Au milieu des années vingt, les réfugiés arméniens manœuvres ou maçons se sont peu à peu dirigés vers le commerce d'articles de confection cousus par les femmes et les enfants puis vont développer un artisanat de chaussures et surtout de bijoux et étendre leur activité à la maroquinerie et à l'horlogerie.

Après 1948 et 1967 (guerres israélo-arabes) Bourj-Hammoud accueille des réfugiés palestiniens et surtout des Libanais du sud. Arrivent aussi des réfugiés kurdes et syriens à la suite des désordres qui eurent lieu en Irak (révolution de Barzani) et en Syrie (coup d'État).

La transformation de Bourj-hammoud en quartier commerçant va se faire entre 1950 et la fin des années 1960. Au début des années 1970 les Arméniens tiennent des commerces de chaussures, d'articles de confection, des bijouteries et des comptoirs de change. Le quartier comprend plusieurs rues commerçantes spécialisées dans différents secteurs : alimentation, chaussures, cuir, stations d'essence, matériel mécanique, sousagences d'automobile japonaises et françaises, banques (Transorient Bank, Federal Bank, Crédit Populaire). À côté de ce développement du commerce, voisine une vente de sandwichs et de boissons dans des baraques améliorées datant des premières arrivées des Arméniens et qui se transforment la nuit en lieux de jeux. Ce n'est qu'après les années de guerre du Liban que Bourj-Hammoud, qui accueillera des commerçants arméniens chassés du centre de Beyrouth (les déplacés), verra à nouveau son activité de commerce se redéployer. Si aujourd'hui « on trouve de tout » à Bourj-Hammoud, on trouve essentiellement des produits qui sont importés de Taïwan et de Chine dans de vastes magasins (les enseignes, 1 \$ SHOP ou 500 L.L. Big solde, avertissent le passant) comme dans des boutiques d'appareils photos numériques, de portables et matériel HiFi etc. Au début de la rue d'Arménie la «Western Union» avec sa devanture jaune et noire où l'on peut lire "the fastest way to send and receive money worlwide " répond aux propos d'un vieux tailleur arménien «ce quartier c'est comme en Amérique, il y a beaucoup d'étrangers ». Et juste à côté, des commerces indiens et sri lankais, proposent des cassettes de musique indienne, des textiles indiens, des produits d'épicerie, alors qu'à l'étage supérieur, le « pamma mini market » offre une restauration indienne. Depuis une dizaine d'années ce sont bien ces migrants qui se sont inscrits dans le paysage de Bourjhammoud ; habitants du quartier ou de passage sont devenus des acteurs de la diversité sociale, économique et professionnelle.

29 Nombre de ces migrants sont en situation illégale (3\% selon la police; chiffre fortement sous-évalué selon d'autres sources) et occupent des emplois précaires: domestiques, employés dans les métiers du bâtiment, employés dans des magasins (coursiers) ou dans des restaurants (serveurs, cuisiniers, plongeurs, nettoyage...), employés dans l'industrie (charcuterie, fabrication de la bière Almaza, fabrique de fours à pain exportés dans les pays arabes) ouvriers dans des fabriques de chaussures, de vêtements de cuir, confection, et de sacs plastiques et enfin employés dans des garages. 


\section{La rue d'Arménie} concentration des échanges économiques et commerciaux. Les marques qu'impriment les Sri lankais, Indiens, Philippins à l'espace de la rue sont visibles, témoignages des changements que connaît Bourj-Hammoud, quartier où les commerçants arméniens restent dominants. La rue d'Arménie est diversement fréquentée et utilisée : espace ouvert où l'on s'active et se montre apprêté le dimanche, jour de repos et d'approvisionnement, espace des hommes du quartier, installés aux terrasses des bistrots-trottoirs, espace des migrants aussi qui s'approvisionnent dans les magasins indiens ou sri lankais et les commerces-trottoirs où l'on peut acheter des cassettes d'artistes indiens. Mais aussi, espace des transactions monétaires que les migrants opèrent dans les boutiques de transferts de fonds, tenues par des Libanais dont les employés traitent dans les différentes langues des clients (anglais, hindi, singhalais...). Les « nouveaux » commerçants se sont insérés dans ce tissu commercial à la faveur de contrats informels passés avec des propriétaires libanais (le montant de la location est de l'ordre de 7000 dollars par an déclarés par les propriétaires) et s'approvisionnent indifféremment dans les pays d'origine ou chez un grossiste spécialisé dans l'import de marchandises en provenance d'Inde et du Sri Lanka. Aujourd'hui, une dizaine de ces magasins-restaurants peuvent offrir des produits qui répondent aux demandes des migrants: cassettes de chanteurs indiens et sri lankais, épices, primeurs, petite épicerie, restauration et bijouterie. La rue d'Arménie attire aussi une clientèle de migrants employés dans le Beyrouth-centre, qui le dimanche viennent remplir leur journée, occupés à consommer, téléphoner à la famille des cabines nichées devant les bureaux de change. À l'heure du repas les restaurants indiens, sri lankais et philippins (quelques restaurants, épiceries se sont installés dans les rues adjacentes à la rue d'Arménie) reçoivent les Philippines qui ont assisté à un office dans une salle transformée en église à l'étage d'un centre associatif géré par des sœurs libanaises ou les Indiens qui se sont retrouvés dans un espace de réunion pour pratiquer leur religion, le bouddhisme.

Que les sociabilités dans la rue d'Arménie ne se résument qu'à une co-présence de tous ceux qui la fréquentent ne fait guère de doute, même si quelques rares couples mixtes marchent côte à côte. Reste que si les habitants du quartier disent que ces populations «ne gênent " pas, ils constatent qu'après le départ des Syriens une autre "clientèle » est en mesure d'occuper les appartements que les classes moyennes désertent. Ils s'arrangent aussi d'une "clientèle » qui reprend des commerces que les Arméniens délaissent : contrats en parole que le «Kalif» (le garant qui est aussi le propriétaire des murs) peut ne pas respecter. Guest Workers, en transit, les migrants sont perçus comme de passage, et pourtant il n'est pas rare de rencontrer des Philippines par exemple qui travaillent ici depuis une dizaine d'années et qui ont aménagé leur vie entre le travail ici et le temps des retrouvailles avec la famille là-bas.

Les Philippines que l'on rencontre le dimanche, rue d'Arménie dans des magasins ou dans une agence de transfert d'argent ou chez un photographe ou encore chez un bijoutier se retrouvent à Bourj-Hammoud pour quelques-unes dans un restaurantépicerie tenu par un Libanais à la retraite. Celle que l'on appelle "la patronne", migrante philippine, fait la cuisine et conseille sur les produits à base de plantes fabriqués en Chine qu'achètent ses compatriotes en grande quantité pour les revendre 
au pays quand elles y retournent. Avant de venir au Liban elle a travaillé à Hong Kong. Après avoir été domestique pendant deux ans dans une famille libanaise, elle a réussi à «s'émanciper » du sponsor pour travailler en free lance. Elle envoie régulièrement de l'argent à ses cinq enfants dont deux sont mariés. Son amie fait des ménages à l'heure après avoir travaillé dans un hôtel. Ces trois enfants sont au pays. Toutes les deux sont en règle mais ce n'est pas le cas de bien d'autres qui se sont retrouvées sans passeports après avoir fui leurs employeurs et qui travaillent à l'heure chez des particuliers (Jureidini, 2003). D'autres encore rencontrées dans un centre de la Caritas trouvent là de l'aide et un espace de prière. Celles qui sont « autonomes » professionnellement (qui ne dépendent pas d'un kalif ou d'un sponsor), vivent en général à plusieurs dans le sud du quartier là où les appartements sont le moins cher. Toutes ces femmes circulent dans le quartier et font en quelque sorte leur propre travail de terrain pour trouver du travail, entrer en relation, chercher aide et soutien, s'allier avec des compatriotes pour constituer des espaces de sociabilités. Si leur présence ne va pas de soi («les immigrés sont tolérés mais pas acceptés, ils ont mis des problèmes dans la société libanaise 》 selon les propos d'un commerçant libanais) leur présence se fait de moins en moins déplacée.

De façon générale à Bourj-Hammoud les migrants ne sont pas protégés du racisme, de l'exclusion ou du rejet, encore moins du contrôle policier, mais se crée dans cette banlieue en construction un monde social où les migrants ajoutent aux jeux complexes des petites solidarités de groupements, de communautés et de confessions leurs propres jeux.

\section{Neve Sha'anan, à l'écart ba'olam}

Longtemps pensé comme hors du monde (ba'olam), la société israélienne compose aujourd'hui l'une des «formes limites» de la mise en présence qu'organise la mondialisation migratoire et offre une lecture empirique parallèle à la situation libanaise.

Ancré depuis la fin du XIX ${ }^{e}$ siècle dans un particularisme migratoire qui a fait d'Israël un État dont la seule source de croissance démographique reposait sur les migrations juives, inscrites dans une idéologie nationale mêlant modernité politique et religieuse, les années quatre-vingt-dix sont venues rompre avec les fondamentaux de la construction nationale. L'échec des négociations de paix avec les Palestiniens et l'inscription affirmée du pays dans la mondialisation des économies ont mené Israël vers une transition socio-politique qui a vu le pays accueillir une présence étrangère de plus en plus nombreuse. Qu'elle soit entrée comme main-d'œuvre de substitution dans le cadre de contrats de travail autorisés pour remplacer les travailleurs palestiniens interdits d'accès à leurs lieux d'activités en Israël ou bien de manière détournée via un visa touristique, cette population de migrants internationaux (estimée globalement à plus de 200000 personnes) est venue s'inscrire dans le débat de société perturbant ainsi le caractère immuable de l'idéologie nationale.

$\mathrm{Au}$ fil des années, la présence des travailleurs étrangers s'est faite perceptible. Si dans les premiers temps, la figure de l'immigré est restée celle d'une femme asiatique poussant une personne âgée sur son fauteuil roulant, progressivement cette image a évolué pour devenir multiple. Pour reprendre les termes d'Arjun Appaduraï (1990), l'expérience que connaît Israël sur cette période consiste en un bouleversement de l'ethnoscape israélien. Des moshavim du Nord aux quartiers centraux des grandes cités 
israéliennes, les travailleurs étrangers ont inscrit leur présence dans le paysage social du pays. Condition sine qua non de l'urbain (Brody, 2005 :11), la rue devient alors l'espace d'une mise en visibilité de l'ouverture au monde induite par cette nouvelle immigration et le moyen de saisir la réalité de la globalisation.

Guidés avant tout par le souci de trouver un espace d'installation correspondant à leurs revenus, les travailleurs étrangers se sont installés dans les quartiers pauvres de Tel Aviv, du fait de la concentration des emplois dans sa région métropolitaine. Les rues environnantes de la gare routière ont ainsi accueilli une large part de cette population et le quartier est devenu une véritable centralité immigrée. Différentes dynamiques socio-économiques ont présidé à l'émergence de cette centralité. En premier lieu, l'existence d'un parc de logements - frisant pour une large part l'insalubrité - a permis d'inscrire dans le paysage social du quartier cette population qui est venue s'insinuer dans les logements délaissés par une population vieillissante. Au quotidien, la représentation du quartier a évolué. Lors de ses traversées pour se rendre à la gare, la population israélienne s'est habituée à croiser du regard de nouveaux résidents venus d'Europe de l'Est, d'Afrique ou bien encore d'Extrême-Orient. En second lieu, la confirmation de ce lieu en tant que centralité s'est trouvée génératrice d'activités qui ont mis en valeur cette présence immigrée. Certaines sont nées à l'initiative de commerçants qui ont vu dans cette population une source de diversification de leurs activités. De manière similaire aux situations observées dans d'autres quartiers du Moyen-Orient comme celui de Bourj-Hammoud à Beyrouth, des enseignes ont été ouvertes à destination de la population immigrante. Des espaces téléphoniques et des échoppes de transferts de fonds ont jalonné les rues piétonnes et celles attenantes à la gare routière où traditionnellement les commerces de gros ou de produits discount composaient l'essentiel de l'offre disponible. Gérés par des entrepreneurs israéliens, les employés sont dans bien des cas issus des pays d'immigration ou sont des olim (immigrants juifs) d'ex-URSS qui valorisent leurs compétences linguistiques dans des activités dédiées aux travailleurs venus d'Europe centrale et orientale. Dans les interstices laissés par les hangars et autres entrepôts, les vitrines de l'industrie du sexe ont également connu une nouvelle jeunesse avec des façades aux offres multilingues redonnant au quartier les tonalités de ses années soixante-dix lorsqu'il était l'espace d'implantation d'immigrants venus principalement du Maghreb. De nombreuses agences immobilières - pour certaines semblant presque improvisées - ont émergé dans le paysage du quartier avec la particularité de ne laisser aucune ambiguïté sur la clientèle attendue. En parallèle et sur l'initiative des immigrants, l'espace public est également devenu le lieu de marchés aux puces où la main-d'œuvre étrangère a pu se fournir en produits déclassés et autres produits de seconde main.

L'ensemble de ces activités a profondément modifié l'image du quartier. S'opposant à la grisaille qui le caractérisait jadis, de nombreux commerces aux noms évocateurs (Disco Bucovina, Mac China etc.) animent aujourd'hui les rues et lui redonnent un dynamisme populaire notamment par la juxtaposition de cafétérias aux terrasses emplies de travailleurs étrangers les yeux rivés sur le téléviseur qui diffuse films et informations dans leur langue. La rue devient alors l'espace d'une communauté de destin où l'expérience de l'autre par "coudoiement » symbolise avec force la mise en présence qu'organise la globalisation (Benayoun, 2005 : 287). L'expérience de l'Autre ne saurait toutefois se résumer à un rapport social dominé par la société majoritaire tant 
l'expérience de la diversité a souligné la porosité des frontières de la société israélienne.

Si les autorités israéliennes ont cherché à réduire la visibilité des travailleurs étrangers, la création de la " police de l'immigration » en septembre 2002 a cependant été un acte de reconnaissance de l'immigration en Israël. Après de longs débats, et surtout grâce à la mobilisation d'associations de défense des travailleurs (notamment Kav La'oved), la situation sociale de cette population n'en a pas pour autant été réduite à une simple question de contrôle des flux comme a pu en témoigner la presse. La question du respect des droits des individus a constitué le premier objectif des articles publiés sur ce thème pour très rapidement souligner le rôle de catalyseur du débat de société que pouvait cristalliser cette population.

Si l'émergence de cette immigration fait espérer à certains que leur pays est sur la voie de la normalisation, d'autres soutiennent que quitter cette voie ne peut mener qu'à la dissolution de l'identité juive de l'État. Ce débat idéologique s'est vu transférer à deux niveaux distincts de la société. En premier lieu, il a concerné l'administration israélienne. L'affirmation de Tel Aviv comme ville d'immigration a mis en opposition le centralisme de l'État et l'administration municipale. La décision de créer un centre d'assistance - nommé Mesila - dédié à l'emploi, l'éducation et la santé en direction des travailleurs étrangers a été la source de tensions entre le gouvernement et les services sociaux de Tel Aviv: le premier dénonçant l'assistance apportée à une population «non-légitime» et les seconds se prévalant d'une mission d'aide aux populations en difficulté (Kemp, 2004 ; Willen, 2003).

En second lieu, c'est dans la société civile qu'un tiers espace a été rendu visible (Bhabha, 1994). Au quotidien, la présence d'immigrés et l'activité commerciale qu'elle a suscitée ont été vécues par un segment - certes minoritaire - de la population israélienne comme l'opportunité d'une transgression. C'est notamment avec le commerce que se donnent à voir ces expériences sociales. Depuis ces trois dernières années, un ensemble de commerces de produits de consommation non-kascher comme des boucheries porcines ont vu le jour. Elles ont pour clientèle la population des travailleurs étrangers des pays de l'Est ou de Chine mais aussi des Israéliens que se définissent comme laïcs et souhaitent intégrer ces nouveaux commerces dans leur quotidien et plus largement dans leur choix de vie. Loin du débat idéologique où s'opposent post et néo-sionistes, la modification du paysage social du quartier et les nouvelles relations sociales qui s'y instaurent sont visibles et ceci malgré la pertinence palpable que semblait avoir (à notre sens) ces changements dans la mesure où personne n'aurait pu imaginer quelques années plus tôt que se seraient installées ces diverses enseignes dont certaines avec des noms comme «The Kingdom of Pork».

42 La relation interculturelle qui existe aujourd'hui à Tel Aviv inscrit l'Autre dans le rapport social. Les clivages sociaux qui traversent la société israélienne ont intégré la présence de ces travailleurs étrangers. Même s'ils doivent demeurer des gastarbeiter pour une large part de la population israélienne et voir leurs droits bafoués, il ressort toutefois que la présence des migrants compose l'un des éléments qui participent au processus de recompositions des frontières sociales dans le pays.

La rue d'Arménie, la gare routière de Tel Aviv ont quelque chose de familier, de déjà-vu. Les situations de co-présence telles que nous les avons observées ne renvoient-elles pas aux situations observées dans d'autres villes de la migration: Milan, Naples, San Diego etc. ? On peut alors s'interroger sur le caractère singulier ou "spécifique " de ces 
observations. Ces «mobilités » transnationales ne s'inscrivent-elles pas là comme dans d'autres régions du monde dans ce qu'il est convenu d'appeler « la mondialisation de la force de travail »? Et ce que l'on appelle aussi les lieux de centralités des côtoiements (gare, rue, banlieue etc.) ne donnent-ils pas à voir « ce qui fait la grande ville d'aujourd'hui, et le manifeste pleinement comme cet espace de "mutuelle étrangeté" et comme ce "siège du cosmopolitisme" que définissait Simmel " (Benayoun, 2005: 282) ? Dès lors ne faut-il pas plutôt voir dans cette "banalité » comment « des occasions sociales » que provoque la rencontre avec l'étranger font événement et de quel sens sont porteuses ces interactions?

\section{Conclusion}

On soulignera pour conclure que l'intérêt qu'il convient d'accorder à la notion de "circulation migratoire» est d'avoir rendu compte de situations et d'expériences diversifiées et d'avoir su imposer une vision dynamique qui considère les circulations comme mouvement entre " deux sédentarités » (Tarrius, 2002). On doit à Gildas Simon d'avoir montré que le mouvement migratoire n'est plus unidirectionnel dans un espace bipolaire, liant départ-arrivée, installation-retour mais pluri-directionnel mettant en relation des espaces. L'interrogation sur les appartenances multiples et les modalités d'identification sont retravaillées dans la perspective de la mise en relation et de la hiérarchisation des lieux par les flux et les relations sociales (multi-appartenance des migrants à des sociétés éloignées). Si l'accent est mis sur les trajectoires et la diversité des parcours, c'est dans la perspective d'analyser « les parcours migratoires » déployés sur des espaces transnationaux.

De l'approche en termes de transnationalisme, on retiendra qu'elle a eu le mérite de bien mettre à jour les questionnements relatifs à la mondialisation migratoire mais tout en voulant s'en éloigner elle reste fortement dépendante de la question de l'assimilation et de l'intégration. Dès lors la tendance à réinterroger à nouveaux frais la question de l'assimilation dans le contexte nord-américain ${ }^{5}$ réinvestit le champ des migrations internationales. Toutefois, le débat aujourd'hui se structure de manière différente et tente de se construire en dehors du couple inclusion/exclusion au national. Comme nous l'avons souligné plus haut, il ne serait être question de définir les appartenances en termes d'identités (infra) nationales puisque les collectifs migrants aspirent à une intégration sociale et économique sans pourtant se définir en référence à la société dans laquelle ils résident et travaillent. La lecture des coprésences que nous avons proposée témoigne de la nécessité de poser le regard en dehors du champ délimité par le devenir du national et de privilégier une démarche où l'observation renseigne sur le quotidien des sociabilités dans l'espace public.

Il convient donc de chercher à saisir dans l'événement de la rencontre le sens des conflits, des ajustements, des petits arrangements et autres accommodements bien plus que de penser les appartenances dans des cadres normatifs. D'autant qu'il n'y a aujourd'hui aucune contradiction à se définir sous les traits d'un " transmigrant " pour reprendre la terminologie des études transnationales tout en investissant l'espace public pour revendiquer le droit à la citoyenneté du « pays d'accueil ». 


\section{BIBLIOGRAPHIE}

APPADURAI Arjun (1990) Disjuncture and difference in the global cultural economy, in M. Featherstone (ed.) Global Culture. Nationalism, Globalization and Modernity, London, Sage Publications, 491p.

BASCH Linda, GLICK-SCHILLER Nina, SZANTON BLANC Christina (1994) Nations Unbound. Transnational projects. Postcolonial Predicaments and Detterritorialized Nations-States, Amsterdam, Gordon and Breach Science Publ., 334 p.

BASCH Linda et al. (Eds.) (1994) Nations Unbound : Transnational Projects, Postcolonial Predicaments, Deterritorialized Nation-states, New York, Gordon and Breach, 344 p.

BENAYOUN Chantal (2005) De la rue ethnique au vaste monde, in J. BRODY (dir.), La rue, Toulouse, Presses Universitaires du Mirail, pp. 281-295.

BHABHA Homi (1994) The Location of Culture, London : Routledge, 285 p.

BRETTELL Caroline (2000) Theorizing Migration in Anthropology, in C. Brettell and J. Hollifield, Migration Theory. Talking across disciplines, New York, Routledge, pp. 97-135.

BRODY J. (dir.) (2005) La rue, Toulouse, Presses Universitaires du Mirail, 311 p.

BUCCIANTI Liliane (1972) Le commerce à Bourj Hammoud, Mémoire de maîtrise, Université SaintJoseph, Beyrouth.

CHIVALLON Christine (1997) De quelques préconstruits de la notion de diasporas à partir de l'exemple antillais, Revue Européenne des Migrations Internationales, vol. 13, n 1, pp. 149-160.

CLIFFORD James (1997) Routes, Travel and translation in the late xxth Century, Cambridge, Harvard University Press, 408 p.

CLIFFORD James (1994) Diasporas, Cultural Anthropology, vol. 9, n 3, pp. 302-338.

COHEN Robin (1997) Global Diasporas : An introduction, London, UCL Press, 228 p.

DE TAPIA Stéphane (1996) Échanges, transports et communications : circulation et champs migratoires turcs, REMI, 12 (2), pp 45-72.

GIDDENS Anthony (2005), La constitution de la société. Éléments de la théorie de la structuration, Paris : PUF (Quadrige), $496 \mathrm{p}$.

GILROY Paul (1994) Diaspora, Paragraph, vol. 17, n 1, pp. 207-212.

GLICK SCHILLER Nina, LEVITT Peggy (2006) Haven't We Heard this Somewher Before? A Substantive View of Transnational Migration Studies by Way of a Reply to Waldinger and Fitzgerald, The Center fo Migration and development, 33 p. (Working Paper Series \#06-01)

GLISSANT Edouard (1997) Traité du tout-monde : Poétique IV, Paris, Gallimard, 261 p.

HANNERZ Ulf (1996) Transnational Connections, Culture People, Places, London-New York, Routledge, $201 \mathrm{p}$.

HILY Marie-Antoinette, DORAI Kamel, MA MUNG Emmanuel (1998) Bilan des travaux sur la circulation migratoire, Migrations Etudes, $\mathrm{n}^{\circ} 84,19 \mathrm{p}$.

HILY Marie-Antoinette, MA MUNG Emmanuel (2003) Catégories et lieux des circulations migratoires, Cahiers de recherches de la MIRE, La Documentation Française, n¹6, pp.33-39. 
JACKSON Peter et al. (2004) The spaces of transnationality, in p. Jackson et al., Transnational spaces, London, Routledge, pp. 1-23.

JUREIDINI Ray (2003) L'échec de la protection de l'Etat : les domestiques étrangers au Liban, Revue Européenne des Migrations Internationales, vol. 19, n 3, pp. 95-128.

KEMP Adriana and RAIJMAN Rebecca (2004) « Tel Aviv is not Foreign to You » : Urban Incorporation Policy on Labor Migrants in Israel, International Migration Review, volume 38, Spring, pp. 26-52.

LASSAILLY-JACOB Véronique (1999) Déplacés et réfugiés : la mobilité sous contrainte, Paris : IRD, $504 \mathrm{p}$.

MA MUNG Emmanuel (1998) Groundlessness and Utopia, the Chinese Diaspora and the Territory in E. Sinn (Ed.), The Last Half Century of Chinese Overseas, Hong Kong, Hong Kong University Press, pp. 35-47.

MANRY Véronique (2001) Être en affaire : compétences relationnelles, éthique de la performance et ordre social dans les réseaux commerçants au marché aux Puces de Marseille, in M. Peraldi, Cabas et containers. Activités marchandes informelles et réseaux migrants transfrontaliers, Paris, Maisonneuve et Larose, pp. 279-214.

MONSUTTI Alessandro (2004) Guerres et migrations. Réseaux sociaux et stratégies économiques des Hazaras d'Afghanistan, Paris/Neuchâtel, Ed. de l'Institut d'ethnologie ; Ed. de la Maison des sciences de l'homme, $368 \mathrm{p}$.

PERALDI Michel (dir) (2001) Cabas et containers. Activités marchandes informelles et réseaux migrants transfrontaliers, Paris, Maisonneuve et Larose, 361 p. (Frontières, villes : lieux de passage).

PORTES Alejandro (1999) La mondialisation par le bas. L'émergence des communautés transnationales, Actes de la Recherche en Sciences Sociales, 129, pp. 15-25.

PORTES Alejandro (2001) The debates and significance of immigrant transnationalism, Global Networks, 1 (3), pp. 181-193.

РОтОТ Swanie (2003) Circulation et réseaux de migrants roumains : Une contribution à l'étude des nouvelles mobilités en Europe, Université de Nice Sophia Antipolis.

SCHMOLL Camille (2004) Une place marchande cosmopolite. Dynamiques migratoires et circulations commerciales à Naples, Thèse, Univ. Paris X-Nanterre, 550 p. (sous la dir. de C. Vallat).

SIMMEL Georg, (1999 [1908]), Sociologie. Etudes sur les formes de la socialisation, Paris, PUF, 756 p. (Sociologies)

SIMON Gildas (1995) Géodynamique des migrations internationales dans le monde, Paris : PUF (politique d'aujourd'hui), $429 \mathrm{p}$.

STALKER Peter (2001) The no-nonsense guide to International Migration, Oxford/London : New internationalist-Verso, $114 \mathrm{p}$.

TARRIUS Alain (2002) La mondialisation par le bas, les nouveaux nomades de l'économie souterraine, Paris, Balland, $168 \mathrm{p}$.

WALDINGER Roger, FITZGERALD David (2004) Transnationalism in Question, American Journal of Sociology, 109 (5), pp. 1177-95.

WILLEN S. (2003) Perspectives on Transnational Labour Migration in Israel, Revue Européenne des Migrations Internationales, 19-3, pp. 243-263. 
WIMMER Andréas, GLICK SCHILLER Nina (2002) Methodological nationalism and beyond : nationstate building, migration and the social sciences, Global Networks, 2 (4), pp. 301-334.

\section{NOTES}

1. Le Fonds des Nations Unies pour la Population (FNUAP) définit ces migrants comme toute personne vivant en dehors de son pays de naissance (environ $3 \%$ de la population mondiale).

2. Les "éco-réfugiés " ou réfugiés de l'environnement sont définis comme toute personne qui quitte son « lieu de résidence lorsque l'équilibre population-ressources est durablement rompu ».

3. Cf. l'article de Gildas Simon dans ce même numéro.

4. Voir notamment les travaux de Véronique Manry, Michel Péraldi ou bien encore ceux d'Alain Tarrius.

5. Voir l'article de Roger Waldinger dans ce numéro.

\section{RÉSUMÉS}

L'objectif de cet article est de rendre compte d'une part des usages des notions qui ont été mobilisées pour penser les formes migratoires mondialisées, en rupture avec les approches développées jusque dans les années soixante-dix et d'autre part de privilégier un point de vue qui relève d'un mode d'interrogation qui ne désigne pas tant les causes des migrations ou les motivations à migrer et les transformations qu'elles induisent que d'accéder à une compréhension plus modeste des modes d'organisation des collectifs en co-présence et la façon dont ils saisissent des "occasions ", là où les gens se rencontrent, dans des espaces de sociabilité non figés : la rue d'Arménie dans le quartier de Bourj-Hammoud à l'est de Beyrouth et le quartier de la gare routière à Tel Aviv.

To describe the International Migrations. The experience of co-presence. This article has two primary objectives. The first is to offer an explanation of the functions and notions used to examine forms of international migration, which depart from the approaches developed up until the 1970s. The second is to single out a point of view that is more concerned with gaining better understanding of the organizational modes of collectives in co-presence than about the causes of migrations or the motivation to migrate and the social transformations they lead to. Rather, we examine the manner in which collectives seize "opportunities" when they meet in non set social spaces: La rue d'Arménie in the neighborhood of Bourj-Hammoud in Eastern Beirut and the neighborhood bounding the central bus station in Tel Aviv.

Describir las migraciones internacionales. Las experiencias y la co-presencia. El objetivo de este artículo es exponer los usos de las nociones a las que se ha recurrido para pensar las formas migratorias mundializadas, nociones que rompen con las visiones desarrolladas hasta los años setenta. Se trata, por otro lado, de privilegiar un punto de vista en el que la interrogación hace menos hincapié en las causas, las motivaciones o las transformaciones inducidas por las migraciones. Se propone así acceder a una comprensión más modesta de los modos de organización de los colectivos en co-presencia y del modo en que dichos colectivos aprovechan las « ocasiones » allí donde la gente se da encuentro, en espacios de sociabilidad no fijos : la calle 
de Armenia del barrio Bourj-Hammoud del Este de Beirut y el barrio de la estación de autobús de Tel-Aviv.

INDEX

Mots-clés : circulation migratoire, commerce, méthodologie, recherche, ville

Index géographique : Israël, Liban

\section{AUTEURS}

WILLIAM BERTHOMIÈRE

Chargé de recherches, Migrinter UMR 6588, MSHS, 99 avenue du recteur Pineau, 86000 Poitiers.

\section{MARIE-ANTOINETTE HILY}

Chargée de recherches, Migrinter UMR 6588, MSHS, 99 avenue du recteur Pineau, 86000 Poitiers. 